Bull. Austral. Math. Soc.

Vol. 74 (2006) [443-448]

\title{
PACKING DIMENSION AND MEASURE OF HOMOGENEOUS CANTOR SETS
}

\author{
H.K. BAEK
}

\begin{abstract}
For a class of homogeneous Cantor sets, we find an explicit formula for their packing dimensions. We then turn our attention to the value of packing measures. The exact value of packing measure for homogeneous Cantor sets has not yet been calculated even though that of Hausdorff measures was evaluated by $Q u, R a o$ and $S u$ in (2001). We give a reasonable lower bound for the packing measures of homogeneous Cantor sets. Our results indicate that duality does not hold between Hausdorff and packing measures.
\end{abstract}

\section{INTRODUCTION}

From the wide variety of fractal dimensions in use, the definition of Hausdorff measures, based on a construction of Caratheodory, is the oldest and probably the most important. The Hausdorff dimension has the advantage of being defined for any set and is mathematically convenient as it is based on measures which are relatively easy to manipulate. Packing measures and dimensions, introduced by Tricot[8], are much more recent. Their similarities to and differences from Hausdorff measures and dimensions are providing an important theoretical tool. The introduction of packing measures has led to a greater understanding of the geometric measure theory of fractals ([7]), with packing measures behaving in a way that is dual to Hausdorff measures in many respects.

One of the disadvantages of studying fractal measures and dimensions ( Hausdorff and packing measures ) is that in many cases they are hard to calculate or estimate computationally. However, many researchers $[1,3,4,5,6,9,10]$ have found exact values or estimated the lower and upper bounds of measures and dimensions for some fractal sets. In these papers and the references therein the interested reader can find an analysis of the exact dimension and measure of some particular fractal sets. In particular, explicit formulae for Hausdorff dimensions of specific Cantor sets were found in $[4,5,6]$, while in $[\mathbf{1}, \mathbf{3}, \mathbf{9}, \mathbf{1 0}]$ exact values were calculated for Hausdorff measures for some Cantor sets, including homogeneous Cantor sets.

This paper analyses the behaviour of the packing dimensions and measures on a homogeneous Cantor set $E$, which is a generalised form of self - similar Cantor sets. We

Received 26th June, 2006

Copyright Clearance Centre, Inc. Serial-fee code: 0004-9727/06 \$A2.00+0.00. 
need Baire category theory and the mass distribution principle to get the exact value of the packing dimension of a set $E$. To obtain a lower bound of packing measure, we use the pseudo-packing measure first adopted by Raymond and Tricot [7].

We define the packing measure and dimension as follows:

A $\delta$-packing of $E \subset \mathbb{R}^{n}$ is a family of pairwise disjoint balls with centre in $E \subset \mathbb{R}^{n}$ and diameter less than or equal to $\delta$. The s-dimensional pre-packing measure of $E \subset \mathbb{R}^{n}$ is

$$
P^{s}(E)=\lim _{\delta \rightarrow 0} \sup \left\{\sum_{i=1}^{\infty}\left|U_{i}\right|^{s}:\left\{U_{i}\right\}_{i=1}^{\infty} \text { is a } \delta \text {-packing of } E\right\},
$$

where $|U|$ is the diameter of a subset $U$ in $\mathbb{R}^{n}$. The s-dimensional packing measure of $E \subset \mathbb{R}^{n}$ is

$$
p^{s}(E)=\inf \left\{\sum_{i=1}^{\infty} P^{s}\left(E_{i}\right): E \subset \cup E_{i}\right\} .
$$

We define the packing dimension in the usual way:

$$
\operatorname{dim}_{\mathrm{p}}(E)=\sup \left\{s: p^{s}(E)=\infty\right\}=\inf \left\{s: p^{s}(E)=0\right\} .
$$

Homogeneous Cantor sets are nowhere dense perfect subsets of $[0,1]$ constructed in the following manner $([4,9])$. Suppose $I=[0,1]$ and let $\left\{n_{k}\right\}_{k \geqslant 1}$ be a sequence of positive integers and $\left\{r_{k}\right\}_{k \geqslant 1}$ a real number sequence satisfying $n_{k} \geqslant 2,0<n_{k} r_{k}<1(k \geqslant 1)$. For any $k \geqslant 1$, let

$$
D_{k}=\left\{\left(i_{1}, \ldots, i_{k}\right): 1 \leqslant i_{j} \leqslant n_{j}, 1 \leqslant j \leqslant k\right\}, \quad D=\bigcup_{k \geqslant 0} D_{k},
$$

where $D_{0}=\phi$. If

$$
\sigma=\left(\sigma_{1}, \ldots, \sigma_{k}\right) \in D_{k}, \tau=\left(\tau_{1}, \ldots, \tau_{m}\right) \in D_{m}
$$

Let

$$
\sigma * \tau=\left(\sigma_{1}, \ldots, \sigma_{k}, \tau_{1}, \ldots, \tau_{m}\right) .
$$

Let $\mathcal{F}=\left\{I_{\sigma}: \sigma \in D\right\}$ be the collection of closed sub-intervals of $I$ which satisfy

(i) $I_{\phi}=I$;

(ii) For any $k \geqslant 1$ and $\sigma \in D_{k}, I_{\sigma * i}\left(1 \leqslant i \leqslant n_{k}\right)$ are sub-intervals of $I_{\sigma}$. $I_{\sigma * 1}, \ldots, I_{\sigma * n_{k}}$ are arranged from the left to the right, $I_{\sigma * i}$ and $I_{\sigma}$ have the same left endpoint, $I_{\sigma * n_{k}}$ and $I_{\sigma}$ have the same right endpoint, and the lengths of the gaps between any two consecutive sub-intervals are equal. We denote the length of one of the gaps of $d_{k}$.

(iii) For any $k \geqslant 1$ and $\sigma \in D_{k}, 1 \leqslant j \leqslant n_{k}$, we have

$$
\frac{\left|I_{\sigma * j}\right|}{\left|I_{\sigma}\right|}=r_{k}
$$

where $|A|$ denote the diameter of $A$. 
Let $E_{k}=\bigcup_{\sigma \in D_{k}} I_{\sigma}, E=\bigcap_{k \geqslant 0} E_{k}$. We call $E$ the homogeneous Cantor set determined by $\left\{n_{k}\right\}_{k \geqslant 1}\left\{r_{k}\right\}_{k \geqslant 1}$ and call $\mathcal{F}_{k}=\left\{I_{\sigma}: \sigma \in D_{k}\right\}$ the $k$ th - order basic intervals of $E$. The middle-third Cantor set and the symmetric perfect set $([5,10])$ are well-known examples of homogeneous Cantor sets.

\section{PACKINg Dimension of homogeneous CANTOR SETS}

In this section, we express the packing dimension of a homogeneous Cantor set as the explicit form with $n_{k}$ and $r_{k}$. First, we define an auxiliary dimension set function by $q^{s}(E)=\varlimsup_{m \rightarrow \infty} \prod_{k=1}^{m} n_{k} r_{k}^{s}$ for $s \geqslant 0$. This function gives a number

$$
\operatorname{dim}_{\mathrm{q}}(E)=\inf \left\{s \geqslant 0 \mid q^{s}(E)=0\right\}=\sup \left\{s \geqslant 0 \mid q^{s}(E)=\infty\right\}
$$

THEOREM 2.1. Let $E$ be a homogeneous Cantor set. Then $\operatorname{dim}_{\mathrm{p}}(E) \geqslant \operatorname{dim}_{\mathbf{q}}(E)$. Proof: If $\operatorname{dim}_{\mathrm{q}}(E)>t$, then $q^{t}(E)=\infty$, that is, $\varlimsup_{m \rightarrow \infty} \prod_{k=1}^{m} n_{k} r_{k}^{t}=\infty$.

Let $\delta>0$ be given. Take $k$ with $\left|I_{\sigma}\right|<\delta$ for all $\sigma \in D_{k}$. Then, for $\sigma \in D_{k}$,

$$
\begin{aligned}
p_{\delta}^{t}\left(I_{\sigma} \cap E\right) & \geqslant \sup _{m \geqslant k} \sum_{I_{+} \subset I_{\sigma}, \tau \in D_{m}}\left|I_{\tau}\right|^{t}=\left|I_{\sigma}\right|^{t} \sup _{m \geqslant k} \sum_{I_{+} \subset I_{\sigma}, \tau \in D_{m}} \frac{\left|I_{\tau}\right|^{t}}{\left|I_{\sigma}\right|^{t}} \\
& =\left|I_{\sigma}\right|^{t} \sup _{m \geqslant k} \prod_{l=k}^{m} n_{l} r_{l}^{t} \geqslant\left|I_{\sigma}\right|^{t} \inf _{k} \sup _{m \geqslant k} \prod_{l=k}^{m} n_{l} r_{l}^{t} \\
& =\left|I_{\sigma}\right|^{t} \varlimsup_{m \rightarrow \infty} \prod_{k=1}^{m} n_{k} r_{k}^{t}=\infty .
\end{aligned}
$$

Therefore $p^{t}\left(I_{\sigma} \bigcap E\right)=\infty$ for all $\sigma \in D_{k}$.

Consider $E=\bigcup_{n=1}^{\infty} F_{n}$. Since $E$ is compact, $E=\bigcup_{n=1}^{\infty} \bar{F}_{n}$. By the Baire Category Theorem, we can take $\bar{F}_{n_{0}}$ whose interior in $E$ is not empty. Hence, for sufficiently large $n$, there exist $k$ with $k \geqslant n$ and $\sigma \in D_{k}$ such that $I_{\sigma} \bigcap E \subset \bar{F}_{n_{0}}$. Then

$$
p^{t}\left(F_{n_{0}}\right)=p^{t}\left(\bar{F}_{n_{0}}\right) \geqslant p^{t}\left(I_{\sigma} \cap E\right)=\infty
$$

So $p^{t}\left(F_{n_{0}}\right)=\infty$. Thus we get that $p^{t}(E)=\infty$ and hence $\operatorname{dim}_{\mathrm{p}}(E) \geqslant t$.

Theorem 2.2. Let $E$ be a homogeneous Cantor set with $\inf _{k} r_{k}>0$. Then $\operatorname{dim}_{\mathrm{p}}(E) \leqslant \operatorname{dim}_{\mathbf{q}}(E)$.

Proof: Let $\inf _{k} r_{k}=c(>0)$. Then $r_{k} \geqslant c$ for all $k$.

Suppose $\operatorname{dim}_{\mathrm{q}}(E)<t$. Then $q^{t}(E)=0$ that is, $\varlimsup_{m \rightarrow \infty} \prod_{k=1}^{m} n_{k} r_{k}^{t}=0$. 
Define $\mu\left(I_{\sigma}\right)=1 /\left(n_{1} n_{2} \cdots n_{k}\right)$ for $\sigma \in D_{k}$. We can extend $\mu$ to a mass distribution on $E$. Let $x \in E=\bigcap_{k=1}^{\infty} E_{k}$. We can choose a sequence of intervals $I_{\sigma_{k}}$ satisfying $x \in \bigcap_{k=1}^{\infty} I_{\sigma_{k}}$, where $\sigma_{k} \in D_{k}$. For any $r>0$, we may find $k$ satisfying $\left|I_{\sigma_{k+1}}\right| \leqslant r<\left|I_{\sigma_{k}}\right|$. We have that

$$
\begin{aligned}
& \frac{\mu(B(x, r))}{r^{t}} \geqslant \frac{\mu\left(I_{\sigma_{k+1}}\right)}{\left|I_{\sigma_{k}}\right|^{t}}=\frac{\mu\left(I_{\sigma_{k+1}}\right)}{\left|I_{\sigma_{k+1}}\right|^{t} \frac{\left|I_{\sigma_{k}}\right|^{t}}{\left|I_{\sigma_{k+1}}\right|^{t}}} \\
& =\frac{\mu\left(I_{\sigma_{k+1}}\right)}{\left|I_{\sigma_{k+1}}\right|^{t}\left(\frac{1}{r_{k}}\right)^{t}} \geqslant \frac{\mu\left(I_{\sigma_{k+1}}\right)}{\left|I_{\sigma_{k+1}}\right|^{t}\left(\frac{1}{c}\right)^{t}} \text { (since } r_{k} \geqslant c \text { ) } \\
& =\frac{c^{t}}{n_{1} n_{2} \cdots n_{k+1} r_{1}^{t} \cdots r_{k+1}^{t}}=\frac{c^{t}}{\prod_{l=1}^{k+1} n_{l} r_{l}^{t}} \text {. }
\end{aligned}
$$

So we get

$$
\varliminf_{r \rightarrow \infty}(\mu(B(x, r))) / r^{t} \geqslant \frac{c^{t}}{\varlimsup_{k \rightarrow \infty} \prod_{l=1}^{k+1} n_{l} r_{l}^{t}}=\infty .
$$

Thus, by the mass distribution principle $([2]), p^{t}(E)=0$. Hence $\operatorname{dim}_{\mathrm{p}}(E) \leqslant t$.

We can easily get that

$$
\operatorname{dim}_{\mathrm{q}} E=\varlimsup_{k \rightarrow \infty} \frac{\log n_{1} \cdots n_{k}}{-\log r_{1} \cdots r_{k}}
$$

by elementary calculation. The condition $\inf _{k} r_{k}>0$ leads to an explicit formula with $n_{k}$ and $r_{k}$ of the packing dimension for the homogeneous Cantor sets as follows.

Corollary 2.3. Let $E$ be a homogeneous Cantor set.

(1) $\operatorname{dim}_{\mathrm{p}} E \geqslant \varlimsup_{k \rightarrow \infty}\left(\log n_{1} \cdots n_{k}\right) /\left(-\log r_{1} \cdots r_{k}\right)$

(2) If $\inf _{k} r_{k}>0$ then $\operatorname{dim}_{\mathrm{p}} E=\varlimsup_{k \rightarrow \infty}\left(\log n_{1} \cdots n_{k}\right) /\left(-\log r_{1} \cdots r_{k}\right)$.

EXAMPLE 2.4. We take $n_{k}=2$ for all $k$. Then the homogeneous Cantor set $E$ is a symmetric perfect set $[5,8,10]$ satisfying the assumptions of the Corollary $2.3(2)$. Thus we have $\operatorname{dim}_{\mathrm{p}}(E)=\varlimsup_{k \rightarrow \infty}(k \log 2) /\left(-\log r_{1} \cdots r_{k}\right)$. Kardos [5] calculated the Hausdorff dimension as $\operatorname{dim}_{\mathrm{H}}(E)=\varliminf_{k \rightarrow \infty}(k \log 2) /\left(-\log r_{1} \cdots r_{k}\right)$.

\section{PaCking MEASURE of homogeneous CANTOR SETS}

We now evaluate a lower bound of packing measure for a homogeneous Cantor set. To do this, we adopt another definition of packing measure.

A pseudo-packing of a set $E$ is any family $\mathcal{B}$ of bounded subsets of the real line such that, if $B, B^{\prime} \in \mathcal{B}$, then $B^{c} \cap E^{c}=\phi$ and $B \cap B^{\prime} \cap E=\phi$ where $B^{c}$ is the closure 
of $B$. A pseudo-packing $B$ of $E$ by balls centred in $E$ has the following property. If $B_{r}(x), B_{s}(y) \in \mathcal{B}$, then $y \notin B_{r}(x)$. A pseudo-packing gives rise to a pre-measure defined as $R^{s}(E)=\lim _{\delta \rightarrow \infty} R_{\delta}^{s}(E)$ where $R_{\delta}^{s}(E)=\sup \left\{\Sigma_{n}\left|U_{n}\right|^{s}:\left\{U_{n}\right\}\right.$ is a $\delta$ - pseudo-packing of $E$ by symmetric intervals centred in $E$ with radii less than $\delta\}$ and the pseudo-packing measure is

$$
r^{s}(E)=\inf \left\{\Sigma_{k} R^{s}\left(E_{k}\right): E \subset \bigcup_{k} E_{k}\right\}
$$

Raymond and Tricot [7] defined the above measure and showed that for any set $E$, $r^{s}(E)=p^{s}(E)$.

THEOREM 3.1. Let $E$ be a homogeneous Cantor set with $d_{k} \geqslant r_{k} / 2$ and let $s=\operatorname{dim}_{p}(E)$. Then $p^{s}(E) \geqslant 2 \varlimsup_{n \rightarrow \infty} \prod_{k=1}^{n} n_{k} r_{k}^{s}$.

Proof: Since $E$ is a closed, bounded set, $P^{s}(E)=p^{s}(E)=r^{s}(E)=R^{s}(E)$.

For each $I_{\sigma}$, let $I_{\sigma}^{l}$ be the symmetric open interval whose centre is the left endpoint of $I_{\sigma}$ and let $I_{\sigma}^{r}$ be the symmetric open interval whose centre is the right endpoint of $I_{\sigma}$ and whose radius is $\left|I_{\sigma}\right| / 2$. Then $\left|I_{\sigma}^{l}\right|^{s}+\left|I_{\sigma}^{r}\right|^{s}=2\left|I_{\sigma}\right|^{s}$ and $\left\{I_{\sigma}^{l}, I_{\sigma}^{r}\right\}_{\sigma \in D_{n}}$ is a pseudo-packing of $E$ since $d_{n} \geqslant r_{n} / 2$. Let $J_{\sigma}$ denote the gap between two consecutive sub-intervals of order $k+1$. Then $\left|J_{\sigma}\right| \geqslant \max \left\{\left|I_{\sigma, 1}\right| / 2,\left|I_{\sigma, 2}\right| / 2\right\}$ since $d_{k} \geqslant r_{k} / 2$. Thus we have that

$$
\sum_{\sigma \in D_{\mathrm{n}}, I_{\sigma} \mid / 2<\delta}\left(\left|I_{\sigma}^{l}\right|^{s}+\left|I_{\sigma}^{r}\right|^{s}\right) \leqslant R_{\delta}^{s}(E)
$$

So $2 \varlimsup_{n \rightarrow \infty} \prod_{k=1}^{n} n_{k} r_{k}^{s}=2 \varlimsup_{n \rightarrow \infty} \sum_{\sigma \in D_{n}}\left|I_{\sigma}\right|^{s}=\varlimsup_{n \rightarrow \infty} \sum_{\sigma \in D_{n}} 2\left|I_{\sigma}\right|^{s} \leqslant R^{s}(E)$.

Therefore $2 \varlimsup_{n \rightarrow \infty} \prod_{k=1}^{n} n_{k} r_{k}^{s} \leqslant p^{s}(E)$.

EXAMPLE 3.2. For the middle-third Cantor set, we have $p^{s}(E) \geqslant 2$ from Theorem 3.1 since $\varlimsup_{n \rightarrow \infty} \prod_{k=1}^{n} n_{k} r_{k}^{s}=1$. But it is shown in [3] using density theory that $p^{s}(E)=4^{s}>2$, where $s=\log 2 / \log 3$. It is also well known that $H^{s}(E)=1$.

EXAMPLE 3.3. If we apply Theorem 3.1 to example 2.4, we get $p^{s}(E) \geqslant 2 \varlimsup_{n \rightarrow \infty} 2^{n} \prod_{k=1}^{n} r_{k}^{s}$, where $s$ is the packing dimension of $E$. It follows from [9] that the Hausdorff measure $H^{s}(E)=\varliminf_{n \rightarrow \infty} 2^{n} \prod_{k=1}^{n} r_{k}^{s}$, where $s$ is the Hausdorff dimension of $E$.

We conclude that duality between these two measures does not hold for a homogeneous Cantor set.

\section{REFERENCES}

[1] E. Ayer and R.S. Strichartz, 'Exact Hausdorff measure and intervals of maximum density for Cantor sets', Trans. Amer. Math. Soc. 351 (1999), 3725-3741. 
[2] K.J. Falconer, The geometry of fractal sets : Mathematical foundations and applications (John Wiley and Sons Ltd, New York, 1990).

[3] D-J. Feng, S. Hua and Z-Y. Wen, 'The pointwise densities of the Cantor measure', J. Math. Anal. Appl. 250 (2000), 692-705.

[4] D-J. Feng, H. Rao and Z-Y. Wen, "The net measure properties of one dimensional homogeneous Cantor set and its applications', Progr. Natur. Sci. 6 (1996), 673-678.

[5] J. Kardos, 'Haudorff dimension of symmetric perfect sets', Acta Math. Hungar. 84 (1999), 257-266.

[6] L. Olsen, 'The exact Hausdorff dimension functions of some Cantor sets', Nonlinearity 16 (2003), 963-970.

[7] X.S. Raymond and C. Tricot, 'Packing regularity of sets in n-space', Math. Proc. Cambridge Philos. Soc. 103 (1988), 133-145.

[8] C. Tricot Jr., 'Two definitions of fractional dimension', Math. Proc. Cambridge Philos. Soc. 91 (1982), 57-74.

[9] C.Q. Qu, H. Rao. and W.Y. Su, 'Hausdorff measure of homogenous Cantor set', Acta Math. Sin. (Engl. Ser.) 17 (2001), 15-20.

[10] C.Q. Qu, Z.L. Zhou and B.G. Jia, 'The upper densities of symmetric perfect sets', $J$. Math. Anal. Appl. 292 (2004), 23-32.

Department of Mathernatics

Kyungpook National University

Daegu 702-701 South Korea

e-mail: hkbaek@knu.ac.kr 